\title{
Leader and Junction Processes in the Lightning Discharge as a Source of VLF Atmospherics
}

\author{
Helen R. Arnold and E. T. Pierce \\ Contribution From Stanford Research Institute, Menlo Park, Calif.
}

(Received October 17, 1963; Revised Fcbruary 17, 1964)

\begin{abstract}
Amplitude spectra are deduced for trains of leader pulses and of $K$ pulses from assumed models of the field or current variations in the lightning discharge. A comparison is made with experimental observations. A size distribution of $K$ pulses relative to return stroke pulses is established. Finally a comparison is made between the spectra of atmospherics from leader, $K$ and return-stroke pulses. These peak respectively at 20,8 , and $5 \mathrm{kHz}$, with corresponding relative peak amplitudes of $1: 2: 10$.
\end{abstract}

\section{Introduction}

It is well known that if the electrostatic field change due to a close lightning flash is recorded, the variation in field with time is comparatively smooth [Pierce, 1955a] except for the abrupt change associated with the return stroke of the discharge to earth. Since the electrostatic field varies with $M$, the electric moment, ${ }^{1}$ it might be anticipated that radiated electromagnetic fields (proportional to $\left.d^{2} M / d t^{2}\right)$ would be of significant amplitude only during the return stroke. However, when close records are made with equipment having a high time-resolving capability or under frequency-response conditions that accentuate sudden variations [Kitagawa and Brook, 1960], it is found that rapid changes in $M$ occur during phases of the discharge other than the return stroke. The total variation in $M$ during these rapid changes is small, but is sufficiently fast for $d^{2} M / d t^{2}$ and, in consequence, the radiated fields to be appreciable. It is the purpose of this paper to consider the VLF pulses generated during some of these fast changes. Figure 1 illustrates their relationship to the overall electrostatic variation. The upper curves (a) show the change of field due to a discharge at $100 \mathrm{~km}$ as measured with equipment having a frequency response of say $1 \mathrm{~Hz}-1 \mathrm{kHz}$; the lower curves (b) are for a frequency response of perhaps $1-50 \mathrm{kHz}$. Variations (a) are mostly electrostatic; variations (b) dominantly radiation.

For a cloud discharge, the superimposed pulse structure most important at VLF is associated with so-called $K$ changes; i.e., small rapid field changes. These are generated as a result of the inhomogeneous distribution of charge in the cloud. The $K$ pulses are most pronounced in the later stages of a

1 The electric moment, $M$, of a thundercloud is defined as $2 \Sigma q h$ where $q$ and $h$ are the charge and height respectively of an element of charge, and the summation extends over all the charges associated with the cloud. The quantity $M$ is in common usage among physicists and meteorologists. Radio engineers often prefer to employ "current moment" that is twice (making allowances for the image) the product of the current flowing and the length it energizes. If the height variation is small the current moment is equal to $d M / d t$. cloud discharge; there is evidence that the pulse structure immediately following the initiation of a cloud flash is somewhat different [Kitagawa and Brook, 1960; Ishikawa, 1961].

Well-marked $K$ pulses also occur during the intervals between the return strokes of a flash to earth, and following the final return stroke. For the discharge to ground, the stage preceding the first return stroke is usually known as the leader stage. It also contains superimposed pulses. Those most familiar, which may be termed leader or $L$ pulses, correspond to the luminous steps produced during the progress of an initial leader from the base of the cloud to the ground [Schonland, 1956]. Pulse astructure is also associated with leader development from its initiation within the cloud until its emergence from the base. There are suggestions that this pulse structure does not correspond to true stepping [Clarence and Malan, 1957] and that the latter is primarily a phenomenon of leader progression through free air.

This paper is concerned with the VLF fields associated with the radiation of $K$ and $L$ pulse structure. The following approach is adopted.

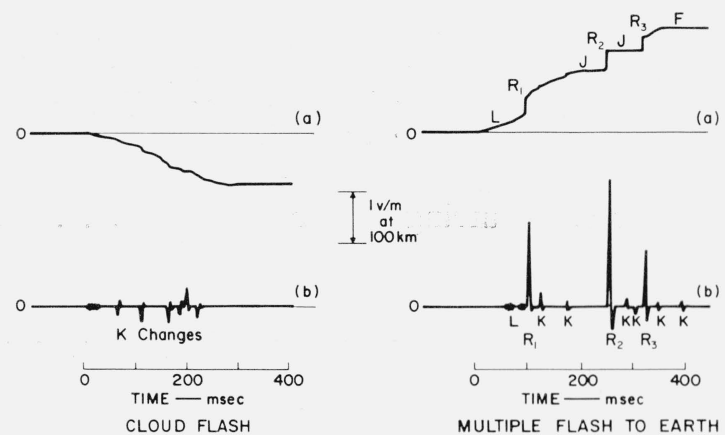

FiguRE 1. Recordings of a typical cloud flash and multiple flash to earth.

(a) $1-1000 \mathrm{~Hz} 100 \mathrm{~km}$ from source, approximately $\sim M$

(b) $1-50 \mathrm{kHz} 100 \mathrm{~km}$ from source, approximately $\sim \frac{d^{2} M}{d t^{2}}$ 
If the total field variation as a function of time $t$ is defined as $E(t)$, then the amplitude spectrum as a function of frequency is given by the modulus of the Fourier transform $F(\omega)$, where $F(\omega)=\int_{-\infty}^{+\infty} E(t) \exp$ $(-i \omega t) \cdot d t$ and $\omega$ is the angular frequency. Then $E(t)$ may be deduced empirically from field observations or derived from estimates of current variations; amplitude spectra can then be obtained. These are compared with observed amplitude spectra, allowances being made, where necessary, for any modifications due to propagation. Since atmospherics are usually recorded at some distance from the flash, this implies an extrapolation back to source conditions.

\section{Leader Pulses}

A lightning flash is initiated by a leader streamer, usually carrying toward the earth negative charge from that accumulated near the base of the thundercloud. It is believed that, having left the cloud, the leader maintains an almost continuous advance toward the ground. However, as regards VLF atmospherics, the superimposed high-current surges that occur, associated with stepping, are more important. Observations of these surges, made photographically by Schonland [1956], suggest that the charge travels about $50 \mathrm{~m}$ in less than a microsecond. The brightness of the channel lasts only a few microseconds, but there is an appreciable pause between surges, lasting between 40 and $100 \mu$ sec. Schonland's observations show a mean pause time $(\bar{\tau})$ between the luminous steps of about $50 \mu$ sec with a standard deviation $\sigma \cong 23 \mu$ sec. A train of leader pulses observed electromagnetically lasts about $3 \mathrm{msec}$ on the average, and contains some 60 pulses. Observations made by Kitagawa [1957] and Kitagawa and Brook [1960] give $\bar{\tau}=70 \mu$ sec with $\sigma=50 \mu$ sec. The intervals between pulses are observed to decrease as the leader approaches the ground [Ishikawa et al., 1958].

If one assumes that the pulses are identical in form and that the pause times are normally distributed about some mean $\bar{\tau}$ with standard deviation $\sigma$, it is possible to derive an expression for the mean amplitude frequency spectrum, which we shall denote by $|L(\omega)|$. The derivation is as follows:

If $E(t)$ is the form of a single pulse and $F(\omega)$ its Fourier transform, then the spectrum of a series of $N$ pulses, occurring at times $T_{1} \cdot T_{2} \ldots T_{n}$ is given by

$$
S(\omega)=\sum_{n=1}^{N} \exp \left(-i \omega T_{n}\right) F(\omega)=F(\omega) \sum_{n=1}^{N} \exp \left(-i \omega T_{n}\right)
$$

The mean power spectrum for such a series is given by $\overline{S(\omega) S^{*}(\omega)}$.

$\overline{S(\omega) S^{*}(\omega)}=F(\omega) F^{*}(\omega) \overline{\sum_{n=1}^{N} \exp \left(-i \omega T_{n}\right) \sum_{n=1}^{N} \exp \left(i \omega T_{n}\right)}$.
Expanding the summations under the bar yields

$$
\begin{aligned}
N & +\sum_{n=1}^{N-1} \exp \left[i \omega\left(T_{n+1}-T_{n}\right)\right]+\exp \left[-i \omega\left(T_{n+1}-T_{n}\right)\right] \\
& +\sum_{n=1}^{N-2} \exp \left[i \omega\left(T_{n+2}-T_{n}\right)\right]+\exp \left[-i \omega\left(T_{n+2}-T_{n}\right)\right]
\end{aligned}
$$

+ further terms

and, writing $T_{n+1}-T_{n}=\bar{\tau}+\epsilon_{n}$, this reduces to

$$
\begin{aligned}
& N+(N-1)\left[\exp (i \omega \bar{\tau}) \overline{\exp \left(i \omega \epsilon_{n}\right.}\right) \\
&\left.+\exp (-i \omega \bar{\tau}) \overline{\exp \left(-i \omega \epsilon_{n}\right)}\right] \\
&+(N-2)\left[\exp (2 i \omega \bar{\tau}) \overline{\exp i \omega\left(\epsilon_{n}+\epsilon_{n+1}\right)}\right. \\
&\left.+\exp (-2 i \omega \bar{\tau}) \overline{\exp -i \omega\left(\epsilon_{n}+\epsilon_{n+1}\right)}\right]
\end{aligned}
$$

+ further terms.

Assuming the $\epsilon_{n}$ all obey the same distribution law and are statistically independent, Large [1957] has shown that

$\overline{\exp i \omega\left(\epsilon_{1}+\epsilon_{2}+\ldots \epsilon_{m}\right)}$

$$
=\left[\int_{-\infty}^{\infty} p(\boldsymbol{\epsilon}) \exp (i \omega \epsilon) d \epsilon\right]^{m}=[P(\omega)]^{m}
$$

where $p(\epsilon)$ is the probability of lying in the range $\epsilon$ to $\epsilon+d \epsilon$. If $N>>1$ and the distribution of $\epsilon_{n}$ is symmetrical about zero, it follows that

$\overline{S(\omega) S^{*}(\omega)}=F(\omega) F^{*}(\omega) N\left[\frac{1-[P(\omega)]^{2}}{1-2 P(\omega) \cos \omega \bar{\tau}+[P(\omega)]^{2}}\right]$

In actuality, the distribution of $\epsilon_{n}$ is skewed with an excess of negative values over that expected from a standard distribution of the $\tau$ 's, but other approximations made in this treatment (e.g., simply the fact that we are deriving an average behavior for all lightning flashes) render the error in this assumption negligible. For a normal distribution of the $\tau_{n}, P(\omega)=\exp \left[-\frac{\sigma^{2} \omega^{2}}{2}\right]$. This gives the mean amplitude spectrum

$$
\begin{aligned}
|L(\omega)|=\left\{S(\omega) S^{*}(\omega)\right\}^{\frac{1}{2}} & \\
& =|F(\omega)| N^{\frac{1}{2}}\left[\frac{\sinh \left(\frac{1}{2} \sigma^{2} \omega^{2}\right)}{\cosh \left(\frac{1}{2} \sigma^{2} \omega^{2}\right)-\cos \omega \bar{\tau}}\right]^{\frac{1}{2}} \\
& =|F(\omega)| N^{\frac{1}{2}} G(\omega) .
\end{aligned}
$$

The form of $G(\omega)$ is shown in figure 2 for three sets of values of $\bar{\tau}$ and $\sigma$. It can be seen that when $\sigma$ is of about the same order of magnitude as $\bar{\tau}$ (as is experimentally observed), $G(\omega)$ does not differ greatly from unity, and hence the spectrum $|L(\omega)|$ of the series of pulses is almost proportional to that $|F(\omega)|$ for the single pulse. However, if $\sigma$ is small, and the stepping has therefore a well-defined repeti- 


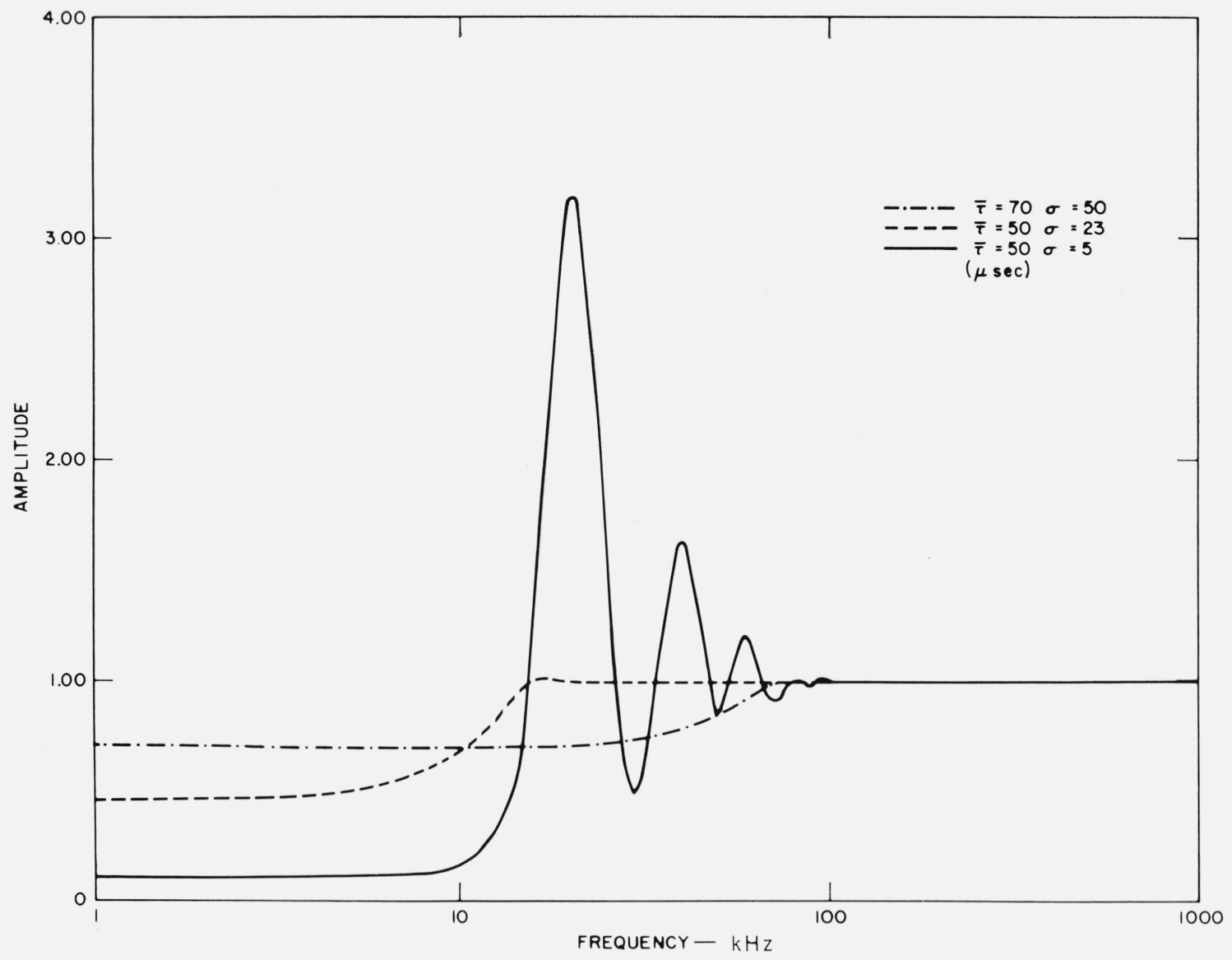

FIgure 2. The function $\mathrm{G}(\omega)$.

tion frequency, $G(\omega)$ has pronounced maxima at the repetition frequency and its harmonics.

As has already been mentioned, the form of $F(\omega)$ depends on the form assumed for $E(t)$. Figure 3 shows two curves derived by the above method and compares them with the form (3) given by Watt and Maxwell [1957] and with an experimental curve derived from the data given in the appendices to Steptoe's thesis [1958]. Curve 1, labeled Pierce, was derived using Schonland's values for $\bar{\tau}$ and $\sigma$, and $N^{\frac{1}{2}}=8$ as suggested by his data. The expression

$$
E(t)=K\left[h_{1}^{3} t \exp \left(-h_{1}^{2} t^{2}\right)+h_{2}^{3} t \exp \left(-h_{2}^{2} t^{2}\right)\right],
$$

as deduced empirically by Pierce [1955a], was used, with $h_{1}=3.5 \times 10^{5} \mathrm{sec}^{-1}$ and $h_{2}=1.2 \times 10^{5} \mathrm{sec}^{-1}$ and, since radiation fields are being considered, $K$ is inversely proportional to distance for short distances from the source; at $100 \mathrm{~km} K=2.7 \times 10^{-12} \mathrm{v} \mathrm{m}^{-1}$ $\sec ^{2}$.

The second curve is derived from the current form for a leader pulse suggested by Steptoe, that is, $I(t)=2 \times 10^{3}[\exp (-\alpha t)-\exp (-\beta t)] \mathrm{A}$, which gives $E(t)=\frac{1}{4 \pi \epsilon_{0}} \cdot \frac{1}{c^{2} d} \cdot 200 \frac{d I}{d t}=4 \times 10^{-7} \quad[-\alpha \exp (-\alpha t)+\beta$ $\exp (-\beta t)]$ at $d=100 \mathrm{~km}$. It is assumed, following Steptoe, that a step of length $100 \mathrm{~m}$ is energized by the current $I(t)$. Steptoe suggests $\alpha=2 \times 10^{4}$ and $\beta=2 \times 10^{5}$ for the parameters. In this model, the current is still appreciably different from zero 50 $\mu$ sec after the pulse begins, which seems unreasonable in the light of Schonland's data.

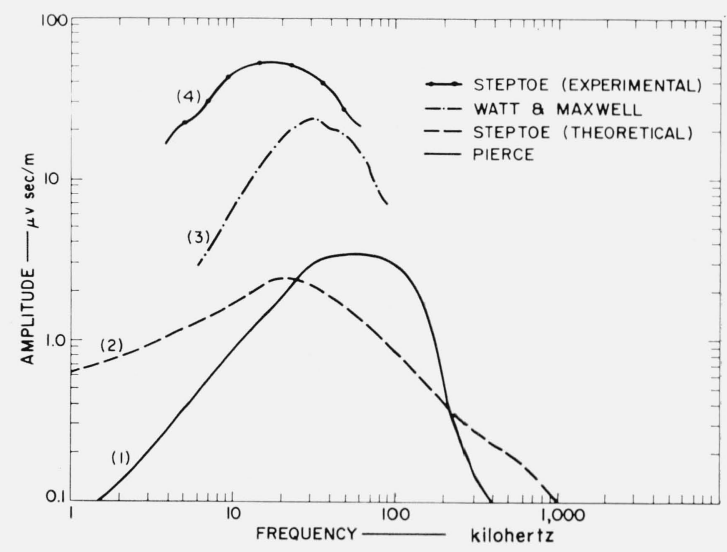

Figure 3. Spectra at $100 \mathrm{~km}$ of a series of leader pulses. 


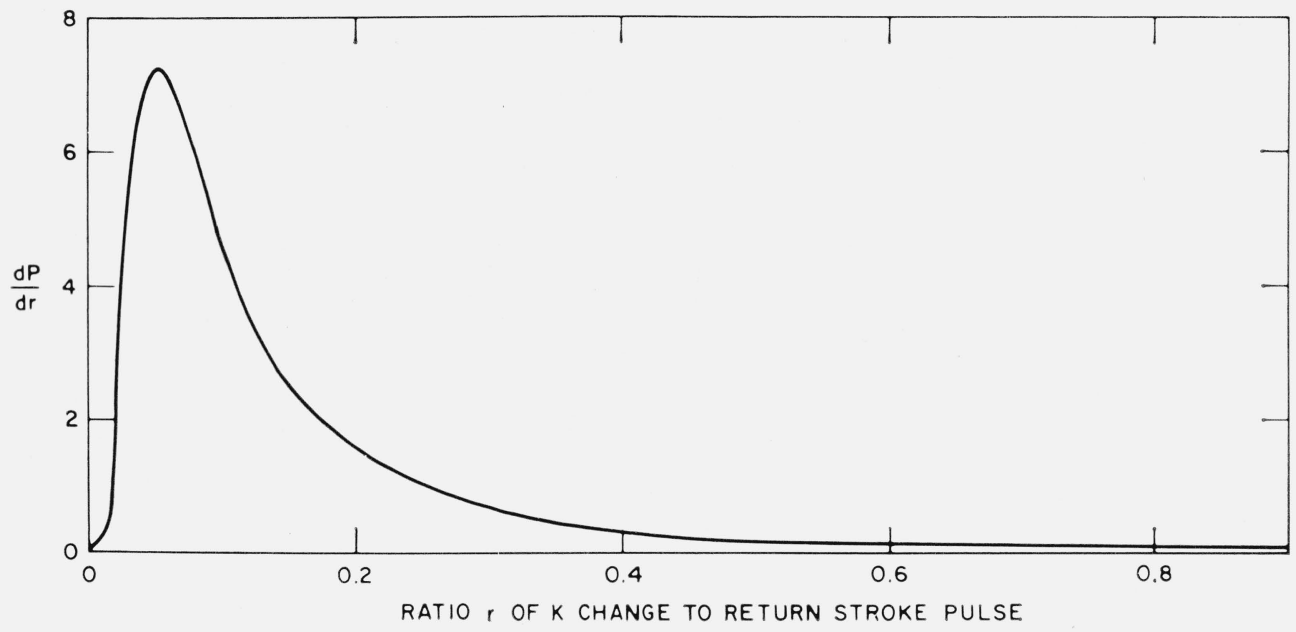

FIGURE 4. Size distribution of K pulses relative to return-stroke pulse.

The discrepancy of about an order of magnitude between the theoretical and the observed results is not entirely unexpected. Steptoe's results were obtained in conjunction with observations by the British CRDF a tmospherics loca ting network [Ockenden, 1947]. With this system, only atmospherics larger than a triggering threshold are fixed. In addition, Steptoe's equipment responded preferentially to atmospherics of large amplitude. It is believed that these experimental conditions imply a bias toward stronger signals. Thus curves 1 and 2 represent average behavior while curve 4 is for the strongest leader disturbances only.

\section{3. $K$ Pulses}

A similar analysis can be applied to the $K$ pulses. The size distribution of these pulses has been experimentally studied by Kitagawa [1962]. His results can be expressed in terms of the ratio $r$ between amplitudes of the $K$ pulses and that of a typical return stroke in a flash to earth. The following empirical relation applies:

$$
\frac{d P(r)}{d r}=\frac{1}{8 \sqrt{2 \pi}} r^{-5 / 2} \exp \left(-\frac{1}{8 r}\right)=F(r)
$$

where $d P(r)$ is the probability of values between $r$ and $r+d r$. This distribution is plotted on figure 4; it gives 0.05 as the most probable value of $r$ and 0.25 as the mean value. This agrees fairly well with the values $0.05 \leq r \leq 0.2$ given by Kitagawa and Brook [1960] and $r=0.1$ given by İshikawa [1961].

The form of a $K$ pulse is not well known, but assuming a double exponential current surge $i(t)=i_{0}$ $[\exp (-\alpha t)-\exp (-\beta t)]$ as suggested by Steptoe [1958] and making the modification that the velocity of advance of the surge along the channel is given by $v(t)=v_{0} \exp (-\gamma t)$ gives

$$
\begin{aligned}
E(t)= & \frac{1}{4 \pi \epsilon_{0}} \frac{1}{c^{2} d} \frac{2 i_{0} v_{0}}{\gamma}[-\alpha \exp (-\alpha t)+\beta \exp (-\beta t) \\
& +(\alpha+\gamma) \exp -(\alpha+\gamma) t-(\beta+\gamma) \exp -(\beta+\gamma) t]
\end{aligned}
$$

Combining the data of Steptoe and Ishikawa [1961], we take $i_{0}=16,000 \mathrm{~A} ; v_{0}=2 \times 10^{7} \mathrm{~m} / \mathrm{sec}, \alpha=5 \times 10^{4}$ $\mathrm{sec}^{-1}, \beta=2 \times 10^{5} \mathrm{sec}^{-1}$, and $\gamma=4 \times 10^{4} \mathrm{sec}^{-1}$. These parameters correspond to a peak current of about 7500 A reached in a time of $9 \mu \mathrm{sec}$; the channel length at the instant of peak current is approximately $150 \mathrm{~m}$, and the asymptotic limit is $500 \mathrm{~m}$.

It is found [Kitagawa and Brook, 1960] that $\bar{\tau}=8.5 \mathrm{msec}$ with $\sigma=6 \mathrm{msec}$ for $K$ pulses. The duration of a series of $K$ pulses depends on the type of flash. The junction stage, between return strokes of a flash to earth, generally lasts about $60 \mathrm{msec}$, while the final stage of such a discharge will continue for about 150 msec. A cloud discharge usually lasts about 250 msec. Thus a series of $K$ pulses may contain anything from 6 to 30 pulses. Since cloud discharges are most common, we shall take $N=25$, giving $N^{\frac{1}{2}}=5$, for this analysis.

The semitheoretical curve thus obtained is plotted on figure 5 and compared with an experimental curve deduced from the data in Steptoe's thesis. Again, the bias due to triggering makes the experimentally measured amplitudes considerably larger than those given by the theoretical curve.

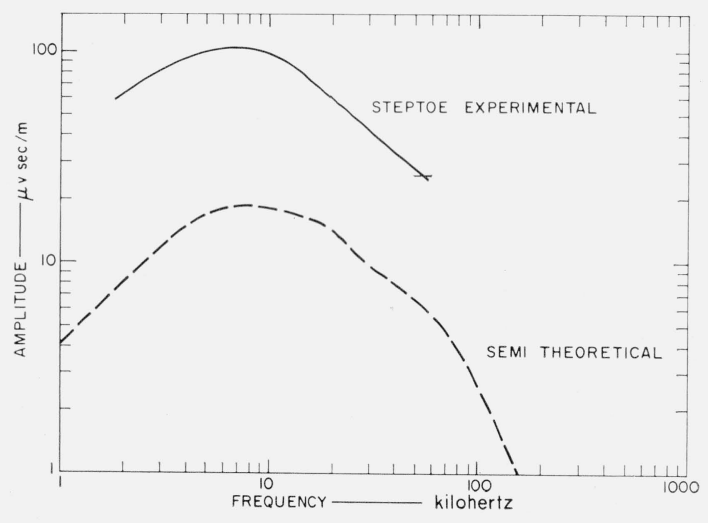

Figure 5. Spectra at $100 \mathrm{~km}$ of a series of $\mathrm{K}$ pulses. 


\section{Discussion}

Amplitude spectra have been deduced for a series of $K$ pulses and for the train of $L$ pulses associated with a stepped leader. In order to relate these to the size of a return-stroke pulse, figure 6 has been prepared. The return-stroke data are obtained from a companion paper [Dennis and Pierce, 1964].

It can be seen that the spectra for a first returnstroke pulse, a series of $K$ pulses, and a series of $L$ pulses have maximum amplitudes in the ratio $10: 2: 1$, occurring at 5,8 , and $20 \mathrm{kHz}$, respectively. Thus, even in the VLF range, the contributions to radio noise from processes other than return strokes are appreciable. This is particularly so for tropical areas, where cloud discharges are perhaps 10 times as frequent as flashes to earth. At high frequency, of course, it is well established [Clarke, 1960] that the return stroke is of little significance in the production of radio noise. Previous work [Pierce, 1955b, 1957; Watt and Maxwell, 1957] has pointed out that the leader can produce appreciable noise at VLF and LF. The present paper shows that the $K$ pulses are considerably more important that the leader disturbance. Within the VLF band, figure 6 indicates that upon the average the signals go in ascending order of magnitude as leader, $K$ change, return stroke. However, the dynamic range at the source is large for all three types of disturbance so that many $K$ changes and even a few leaders are greater than some return strokes. Since $K$ changes occur much more frequently than return strokes their importance as regards VLF noise is evident.

We are indebted to Dr. B. J. Steptoe for being: allowed to use the data contained in his thesis.

This paper summarizes work described in greater detail in a report by Pierce, Arnold, and Dennis [1962] and supported under U.S. Air Force Contract AF 33(657)-7009. We are grateful to the Air Force authorities for permission to publish this paper.as

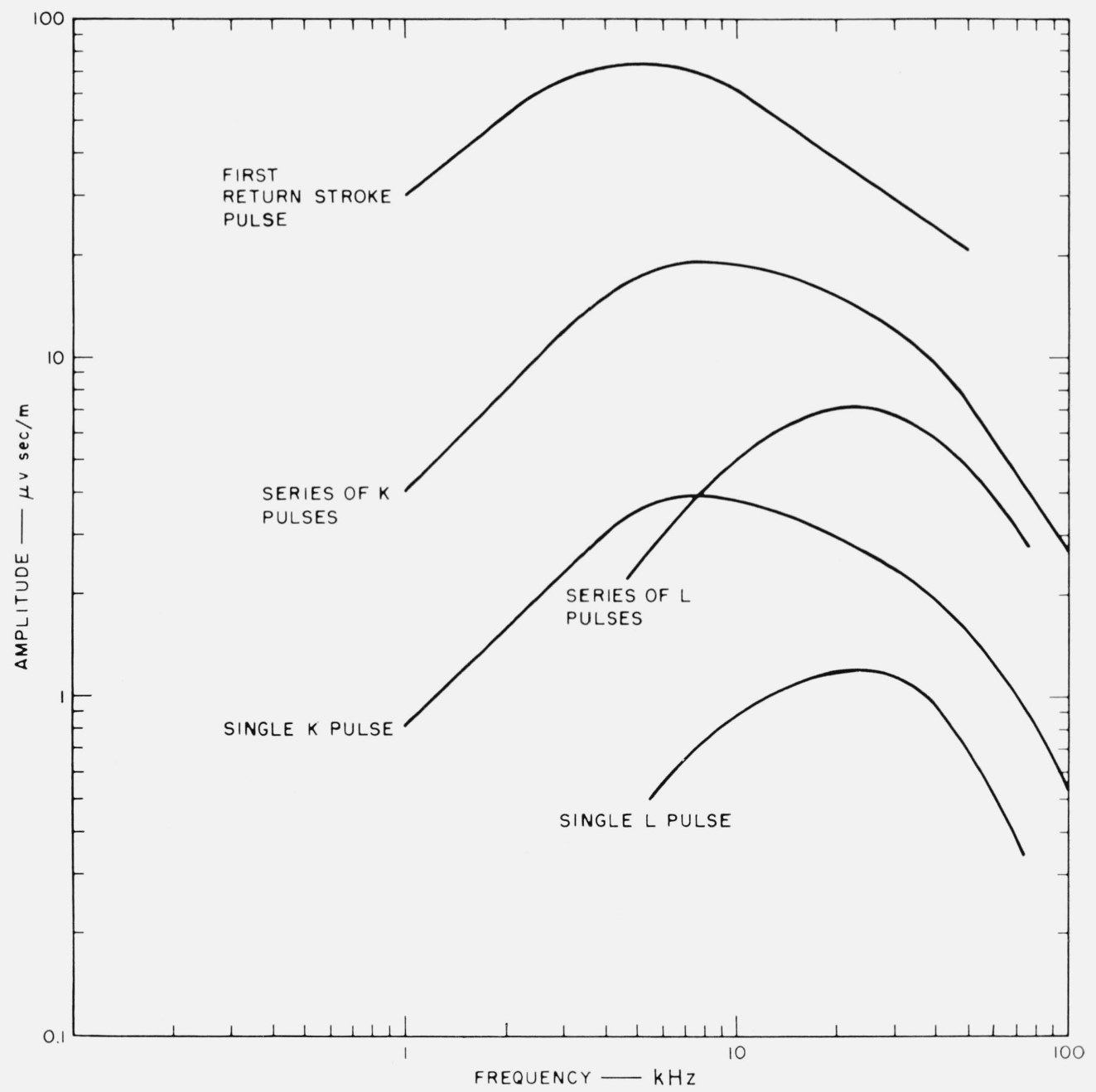

FiguRE 6. Comparison of spectra at $100 \mathrm{~km}$ due to various processes in a lightning flash. 


\section{References}

Clarke, C. (1960), A study of atmospheric radio noise received on a narrow bandwidth at $11 \mathrm{Mc} / \mathrm{s}$, Proc. IEE 10\%B, $311-319$.

Clarence, N. D., and D. J. Malan (1957), Preliminary discharge processes in lightning flashes to ground, Quart. J.

- Roy. Meteorol. Soc. 83, 161-172.

Dennis, A. S., and E. T. Pierce (July 1964), The return stroke of the lightning flash to earth as a source of VLF atmospherics, Radio Sci. J. Res. NBS/USNC-URSI 68D, No. 7, 777 .

Ishikawa, H. (1961), Nature of lightning discharges as origins of atmospherics, Proc. Res. Inst. Atmospherics, Nagoya University $\mathbf{8 A}, 1-247$.

Ishikawa, H., M. Takagi, and T. Takeuti (1958), On the leader waveforms of atmospherics near the origins, Proc. Res. Inst. Atmospherics, Nagoya University 5, 1-11.

Kitagawa, N. (1957), On the electrical field change due to leader processes and some of their discharge mechanisms, Papers, Meteorol. Geophys. (Tokyo) 7, 400-414.

Kitagawa, N. (1962), Private communication.

Kitagawa, N., and M. Brook (1960), A comparison of intracloud and cloud to ground lightning discharges, J. Geophys. Res. 65, 1189-1201.

Large, M. I. (1957), The radio interference produced by corona discharge, J. Atmospheric Terrest. Phys. 10, $240-250$.
Ockenden, C. V. (1947), Sferics, Met. Mag. 76, 78-86.

Pierce, E. T. (1955a), Electrostatic field changes due to lightning discharges, Quart. J. Roy. Meteorol. Soc. 81, 211-228.

Pierce, E. T. (1955b), The frequency spectra of the radiation fields from lighning flashes, Proc. Symposium on VLF Waves and Atmospherics, Munich, Oct. 3, 1955.

Pierce, E. T. (1957), Atmospherics as indicators of worldwide thunderstorm activity. Report of meeting on "Atmospheric Electricity" during the IGY at Aachen May 11/12, 1956, 28-37, ed. H. Israël, Meteorological Observatory, Aachen.

Pierce, E. T., H. R. Arnold, and A. S. Dennis (1962), Verylow-frequency atmospherics due to lightning flashes, Final Report, Contract AF33 (657)-7009, Stanford Research Institute, Menlo Park, California.

Schonland, B. F. J. (1956), The lightning discharge, Handbuch der Physik 22, 576-629.

Steptoe, B. J. (1958), Some observations on the spectrum and propagation of atmospherics, $\mathrm{Ph}$. D. Thesis, University of London, England.

Watt, A. D., and E. L. Maxwell (1957), Characteristics of atmospheric noise from 1 to $100 \mathrm{~km}$, Proc. IRE $\mathbf{4 5}$, $787-794$.

(Paper 68D-374) 